\title{
Effects of local dexmedetomidine administration on the neurotoxicity of ropivacaine for sciatic nerve block in rats
}

\author{
XING XUE $^{1 *}$, JUN FAN $^{2 *}$, XIAOLI MA ${ }^{1}$, YONGQIANG LIU ${ }^{1}$, XUENA HAN $^{1}$, YUFANG LENG ${ }^{1}$ and JINJIA YU ${ }^{3}$ \\ ${ }^{1}$ Department of Anesthesiology, The First Hospital of Lanzhou University, Lanzhou, Gansu 730000; \\ ${ }^{2}$ Department of Anesthesiology, Wuhan Children's Hospital, Tongji Medical College, Huazhong University of Science and \\ Technology, Wuhan, Hubei 430000; ${ }^{3}$ School of Life Sciences, Central South University, Changsha, Hunan 410083, P.R. China
}

Received February 5, 2020; Accepted August 14, 2020

DOI: $10.3892 / \mathrm{mmr} .2020 .11514$

\begin{abstract}
Dexmedetomidine, used as an adjuvant to local anesthetics (LAs), may prolong the duration of peripheral nerve block. However, the effect of dexmedetomidine on the neurotoxicity of LAs is not completely understood. The present study was designed to investigate the efficacy of two doses of dexmedetomidine as an adjuvant to ropivacaine and its protective effect against the neurotoxicity of LAs. Paw withdrawal thermal latency testing was used to detect the sensory blockade. Extensor postural thrust testing was used to detect the motor blockade. The results demonstrated that the addition of dexmedetomidine to ropivacaine prolonged the duration of sensory and motor blockade in a dose-dependent manner compared with ropivacaine alone. TUNEL staining was performed to examine apoptosis. Western blotting was used to detect the Cleaved caspase-3 expression levels. The results showed that the addition of dexmedetomidine to ropivacaine decreased the rate of apoptosis and caspase- 3 expression levels in a dose-dependent manner compared with ropivacaine alone $(\mathrm{P}<0.05)$. In addition, the rate of apoptosis and caspase- 3 expression levels were significantly lower in the high-dose dexmedetomidine group compared with the low-dose dexmedetomidine group $(\mathrm{P}<0.05)$. The results suggested that the addition of dexmedetomidine to ropivacaine for sciatic nerve block in rats not only prolonged the duration of sensory and motor block of the sciatic nerve, but also markedly alleviated ropivacaine-induced neurotoxicity by decreasing caspase-3-dependent sciatic nerve cell apoptosis.
\end{abstract}

Correspondence to: Dr Yufang Leng, Department of Anesthesiology, The First Hospital of Lanzhou University, 1 Donggang West Road, Chengguan, Lanzhou, Gansu 730000, P.R. China

E-mail: lengyf@1zu.edu.cn

"Contributed equally

Key words: nerve block, dexmedetomidine, neurotoxicity, ropivacaine
Furthermore, the present study indicated that dexmedetomidine was more effective at a dose of $20 \mu \mathrm{g} / \mathrm{kg}$ compared with $6 \mu \mathrm{g} / \mathrm{kg}$.

\section{Introduction}

Local anesthetics (LAs) are widely used to relieve acute, intraoperative and postoperative chronic pain (1). Ropivacaine is a novel type of amide LA that blocks fewer motor fibers than sensory fibers, and is less cardiotoxic and neurotoxic compared with other Las (2). Therefore, ropivacaine is commonly selected instead of bupivacaine for postoperative analgesia (3).

The relatively short duration of the effects of a single LA injection frequently leaves the patient in pain when the block wears off, particularly for postoperative analgesia; therefore, prolonging the duration of analgesia is a priority (4). An increase in the dose and/or volume of LA administered may prolong the duration of analgesia but may also increase the risk of neurotoxicity (5). Although continuous catheter-based nerve blocks can extend postoperative analgesia, their placement requires additional time, cost and skill (4).

Several perineural adjuvants have been studied with the goal of prolonging the duration of analgesia, reducing the dose of LA and improving analgesia with fewer adverse effects (6-8). Dexmedetomidine is a potent, highly selective $\alpha_{2}$-adrenoceptor agonist and its $\alpha_{2} / \alpha_{1}$ selectivity is eight times greater than that of clonidine (9). A study reported that dexmedetomidine enhances the duration of bupivacaine anesthesia and analgesia of sciatic nerve block in rats (10). In addition, dexmedetomidine possesses neuroprotective properties in various experimental models (11). LAs are generally thought to be relatively safe and the potential neurotoxicity of LAs has been investigated for a number of years (12). Ropivacaine has been documented to be less cardiotoxic and neurotoxic compared with other Las (13). However, there have been a number of reports on the neurotoxicity of ropivacaine $(14,15)$. LA-induced direct nerve injury can occur following the administration of LAs at clinical concentrations (16), and the mechanism underlying neurotoxicity induced by LAs is complex and not completely understood $(14,17,18)$. Evidence of neuronal apoptosis has been observed in a number of animal models and the mitochondrial pathway has been demonstrated to be involved in LA-mediated apoptosis (17). The degree of 
LA toxicity has been demonstrated to be concentration- or dose-dependent (19).

A previous study has demonstrated that the addition of dexmedetomidine to an LA extends the duration of blockade in the peripheral nerves (20); however, the effect of dexmedetomidine on neurotoxicity is not completely understood. The present study aimed to investigate the effects of dexmedetomidine as an adjuvant to ropivacaine for sciatic nerve block, to explore whether it can mitigate the toxic effect of LAs and to identify the mechanisms underlying dexmedetomidine.

\section{Materials and methods}

Animal selection and housing. The present study was approved by the Ethics Committee of the First Hospital of Lanzhou University (approval no. LDY YLL2019-111)and the procedures were performed according to routine animal care guidelines. Healthy male adult SPF Wistar rats $(n=40$; age 8-10 weeks; weight, 180-220 g) were provided by the Experimental Animal Center of Gansu University of Traditional Chinese Medicine (Lanzhou, China). The rats were housed in separate cages in temperature-controlled rooms $\left(20-24^{\circ} \mathrm{C}\right.$; relative humidity, 50-60\%) with 12-h light/dark cycles, and free access to food and water until the time of experimentation.

Groups and treatment. The rats were randomly divided into the following five groups ( $n=8)$ : i) Control (group C); ii) sham (group S); iii) ropivacaine (group R); iv) low-dose dexmedetomidine group (group D1); and v) high-dose dexmedetomidine group (group D2).

Sciatic nerve block was performed according to the procedure described by Kim et al (21), but in the present study the drug was injected into the perineural space under the fascia covering the nerve. Following anaesthetization with $1.2-1.5 \%$ isoflurane in oxygen using a mask, the bilateral sciatic nerve was exposed by a gluteal muscle-splitting incision. The rats in group $\mathrm{C}$ underwent bilateral sciatic nerve exposure only. The rats in group $\mathrm{S}$ received a bilateral injection of $0.2 \mathrm{ml} 0.9 \% \mathrm{NaCl}$. The rats in group $\mathrm{R}$ received a bilateral injection of $0.2 \mathrm{ml} 0.5 \%$ ropivacaine hydrochloride (batch number: LBDZ; AstraZeneca). The rats in group D1 received a bilateral injection of $0.2 \mathrm{ml} 0.5 \%$ ropivacaine plus $6 \mu \mathrm{g} / \mathrm{kg}$ dexmedetomidine hydrochloride (batch number: 16110732; Jiangsu Hengrui Medicine Co., Ltd.), whereas rats in group D2 received a bilateral injection of $0.2 \mathrm{ml} 0.5 \%$ ropivacaine plus $20 \mu \mathrm{g} / \mathrm{kg}$ dexmedetomidine hydrochloride. A nonabsorbable muscle fascia suture was placed at the midpoint of the injection site as a marker for subsequent nerve removal. Following the injection, the incision was sutured layer by layer, penicillin powder was applied to the surgical wound to prevent infection and the rats were placed into individual feeding chambers.

Paw withdrawal thermal latency $(P W L)$ testing. The time at which the righting reflex returned was recorded to the nearest minute $(0 \mathrm{~min})$ and the rats were then placed in a chamber for the PWL test, which was performed with an RB-200 intelligent hot plate (Shanghai Uilian, Inc.) at $55 \pm 1^{\circ} \mathrm{C}$ every $30 \mathrm{~min}$ for $330 \mathrm{~min}$. The PWL indicated deficiencies in sensory function. The mean value of three measurements at each time point was calculated. A cut-off time of $15 \mathrm{sec}$ was used to avoid tissue damage. If no withdrawal was observed after $15 \mathrm{sec}$, the stimulus was removed and the PWL was recorded as $15 \mathrm{sec}$.

Extensor postural thrust (EPT) testing. To measure the rat hindfoot EPT (22), each rat was held upright with its hindlimb extended such that the body's weight was supported by the distal limb and toes. The extensor thrust, the force that resisted contact of the heel with the platform, was measured as the force applied to the digital platform balance (cat. no. JY303; Shanghai Puchun Measure Instrument Co., Ltd.). A reduction in this force, which represented reduced extensor muscle tone, was considered a deficiency in motor function. Once the sensory and motor function of each rat had returned to baseline, the rat was returned to its home cage.

Histopathological evaluation. For histopathological assessment, at $24 \mathrm{~h}$ post-drug administration, the rats were anaesthetized with $1.2-1.5 \%$ isoflurane and the sciatic nerves were removed from the injection site. Each sciatic nerve was fixed with $10 \%$ formaldehyde solution for $24 \mathrm{~h}$ at room temperature, sequentially dehydrated with grade ethanol $(70,80,95$, 100 and 100\%), cleared in xylene and embedded in paraffin. Sections (thickness, $5 \mu \mathrm{m}$ ) were prepared and deparaffinized at $40^{\circ} \mathrm{C}$ in a water bath and rehydrated. Samples were then washed with distilled water and dried. $2 \%$ hematoxylin was added for $5 \mathrm{~min}$ at room temperature and rinsed with water. Subsequently $1 \% \mathrm{HCl}$ ethanol solution $(1 \mathrm{ml} \mathrm{HCl}$ added to $99 \mathrm{ml} 70 \%$ ethanol) was added for $10 \mathrm{sec}$ in triplicate to remove excess haematoxylin. Following this, sections were washed using distilled water for $25 \mathrm{~min}$, then $0.5 \%$ eosin was added at room temperature for $2 \mathrm{~min}$ and slices were dehydrated with 95 and 100\% ethanol. Dimethylbenzene (Absin Bioscience, Inc.) was added for 5 min twice and incubated at $37^{\circ} \mathrm{C}$ for $24 \mathrm{~h}$. Finally, pathological changes were observed under an optical microscope (magnification, $\mathrm{x} 400$; Olympus Corporation). The rats were euthanized by overdose of isoflurane.

Apoptosis of sciatic nerve cells. TUNEL staining was performed to examine apoptosis. Samples of sciatic nerve were fixed in $10 \%$ formalin for $20 \mathrm{~min}$ at room temperature and embedded in paraffin. Sections $(5 \mu \mathrm{m})$ were deparaffinized, rehydrated and incubated for $15 \mathrm{~min}$ at $37^{\circ} \mathrm{C}$ with proteinase $\mathrm{K}$ working solution (Shanghai Xiangsheng Biotechnology Co., Ltd.). The sections were rinsed twice with PBS (pH 7.4) and incubated in a $0.3 \%$ hydrogen peroxide blocking solution for $15 \mathrm{~min}$ at room temperature. Subsequently, $50 \mu 1$ TUNEL reaction reagent was then added to the sections, which were incubated for $60 \mathrm{~min}$ at $37^{\circ} \mathrm{C}$ in a humidified atmosphere in the dark. Following rinsing with PBS (pH 7.4) three times, $50 \mu 1$ converter peroxidase was added to the sections, which were incubated for $30 \mathrm{~min}$ at $37^{\circ} \mathrm{C}$, and $50 \mu \mathrm{l}$ diaminobenzidine substrate was added to the sections prior to incubation for $10 \mathrm{~min}$ at $25^{\circ} \mathrm{C}$. The sections were rinsed with PBS (pH 7.4) three times and analyzed under a light microscope (magnification, x400). According to the distribution of apoptotic positive cells, three unrepeated visual fields of each section were observed under a light microscope and the percentage of positive cells was calculated as the apoptosis rate (number of apoptotic cells/total number of cells in the field). 
Cleaved caspase-3 expression in the rat sciatic nerve. Cleaved caspase-3 expression was detected via western blotting. The samples were cut into small pieces ( $2 \mathrm{~mm}$ long; $2 \mathrm{~mm}$ thick) and homogenized in lysis buffer $[50 \mathrm{mM}$ Tris $\mathrm{HCl}(\mathrm{pH}$ 7.6), $20 \mathrm{mM} \mathrm{MgCl} 2,150 \mathrm{mM} \mathrm{NaCl}, 0.5 \%$ Triton-X, $5 \mathrm{U} / \mathrm{ml}$ aprotinin, $5 \mu \mathrm{g} / \mathrm{ml}$ leupeptin, $5 \mu \mathrm{g} / \mathrm{ml}$ pepstatin, $1 \mathrm{mM}$ benzamidine, $1 \mathrm{mM}$ phenylmethylsulfonyl fluoride]. Lysate protein levels were determined using a BCA protein assay kit (Shanghai Qcbio Science \& Technologies Co., Ltd.). Equal amounts of protein $(30 \mu \mathrm{g})$ were separated via $10 \%$ SDS-PAGE and subjected to gel electrophoresis. The separated proteins were transferred onto nitrocellulose membranes, which were blocked with 5\% nonfat dried milk in Tris-buffered saline with Tween 20 (137 mM sodium chloride, $20 \mathrm{mM}$ Tris, $0.1 \%$ Tween 20; Absin Bioscience, Inc.) for $1 \mathrm{~h}$ at room temperature. Subsequently, the membranes were incubated with primary antibodies targeted against cleaved caspase-3 (1:1,000; cat. no. bsm-33199M; Beijing Biosynthesis Biotechnology Co., Ltd.) and $\beta$-actin (1:1,000; cat. no. bsm-33036M Beijing Biosynthesis Biotechnology Co., Ltd.) diluted in Tris-buffered saline with Tween 20 overnight at $4^{\circ} \mathrm{C}$. The membranes were then incubated with alkaline phosphatase-conjugated goat anti-mouse IgG secondary antibodies (1:1,000; cat. no. bs-40296G-HRP; Invitrogen; Thermo Fisher Scientific, Inc.) for $1 \mathrm{~h}$ at room temperature and the reactive bands were detected following incubation with nitroblue tetrazolium and 5-bromo-4-chloro-3-indolyl phosphate (Sigma-Aldrich; Merck KGaA) for $5 \mathrm{~min}$ at room temperature. The bands were scanned, their densities were assessed using an imaging densitometer (cat. no. GS-800; Bio-Rad Laboratories, Inc.) and the optical densities were quantified using Image-Pro Plus software (version 7.0; Media Cybernetics, Inc.) with $\beta$-actin as the loading control.

Statistical analysis. Data were statistically analysed using SPSS 20.0 software (IBM Corp.). All data are presented as the mean $\pm(\mathrm{SD})$ of five experimental repeats $(\mathrm{n}=6$ in each group). One-way ANOVA followed by Tukey's post hoc test was performed to determine overall differences in PWLs and EPTs at each time point, as well as the differences in the levels of apoptosis and expression of cleaved caspase-3 among all experimental groups. $\mathrm{P}<0.05$ was considered to indicate a statistically significant difference.

\section{Results}

Neurobehavioral evaluation. Compared with group $\mathrm{R}$, groups D1 and D2 displayed an increased duration of analgesia against a heat stimulus. The time taken to return to baseline sensory function (defined as $\mathrm{P} \geq 0.05$ compared with group $\mathrm{C}$ ) was longer in groups D1 and D2 compared with group R. The time to return to baseline sensory function was longer in group D2 compared with group D1. No significant difference was observed between groups $\mathrm{C}$ and $\mathrm{S}(\mathrm{P}>0.05$; Fig. 1$)$.

Groups D1 and D2 increased the duration of motor blockade compared with group $\mathrm{R}$. The time to return to baseline motor function (defined as $\mathrm{P} \geq 0.05$ compared with group $\mathrm{C}$ ) was longer in groups D1 and D2 compared with group R. The time to return to baseline motor function was longer in group D2 compared with group D1. No significant difference was observed between groups $\mathrm{C}$ and $\mathrm{S}$ ( $\mathrm{P}>0.05$; Fig. 2).

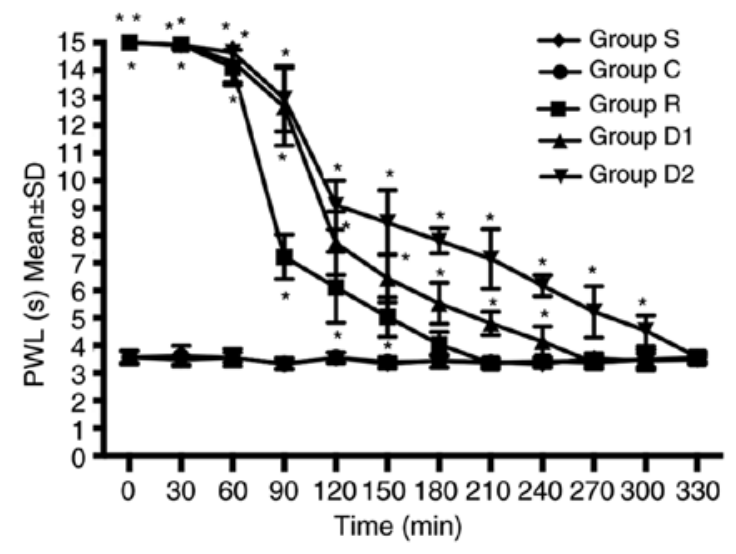

Figure 1. Right hindlimb PWL of the five groups at different time points $(n=8)$. The times required for group $\mathrm{R}$, group D1 and group D2 to return to baseline sensory function were 180,240 and $300 \mathrm{~min}$, respectively. The time to return to baseline sensory function was defined as $\mathrm{P} \geq 0.05$ vs. group $\mathrm{C}$. ${ }^{*} \mathrm{P}<0.05$ vs. group C. PWL (s), paw withdrawal thermal latency (sec); group R, ropivacaine group; group D1, low-dose dexmedetomidine group; group D2, high-dose dexmedetomidine group; group $\mathrm{C}$, control group; group $\mathrm{S}$, sham group.

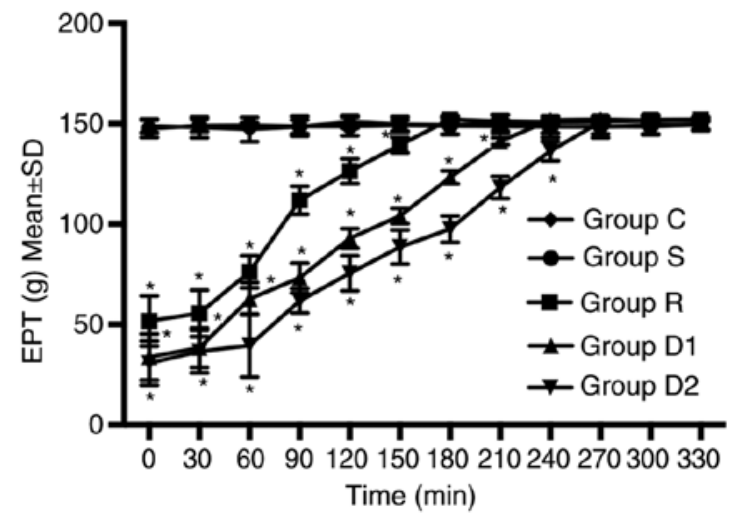

Figure 2. Right hindlimb EPTs of the four groups at different time points $(n=8)$. The times required for group $\mathrm{R}$, group D1 and group D2 to return to baseline motor function were 180,240 and $270 \mathrm{~min}$, respectively. The time to return to baseline motor function was defined as $P \geq 0.05$ vs. group $C$. ${ }^{*} \mathrm{P}<0.05$ vs. group C. EPT, extensor postural thrust; group R, ropivacaine group; group D1, low-dose dexmedetomidine group; group D2, high-dose dexmedetomidine group; group $\mathrm{C}$, control group; group $\mathrm{S}$, sham group.

Histopathologic evaluation. Histopathologic analysis indicated that groups D1 and D2 displayed alleviated nerve injury compared with group $\mathrm{R}$. In groups $\mathrm{S}$ and $\mathrm{C}$, the sciatic nerve was intact, the nerve fibers were arranged tightly and neatly, the morphology was normal, the staining was uniform and the structures of the axons and myelin sheath were clear. In group R, certain sciatic nerve fibers were disordered and interrupted, and certain axons and nerve sheaths displayed edema. Compared with group R, group D1 displayed markedly less disorder and discontinuity in the nerve fiber structure, and axonal and myelin edema in the nerve fibers. Compared with group D1, the fibers of the sciatic nerve in group D2 were arranged more neatly, and myelin sheath and axon edema was milder (Fig. 3).

Alterations in apoptosis. The TUNEL assay was used to observe neuronal apoptosis under a light microscope. The 


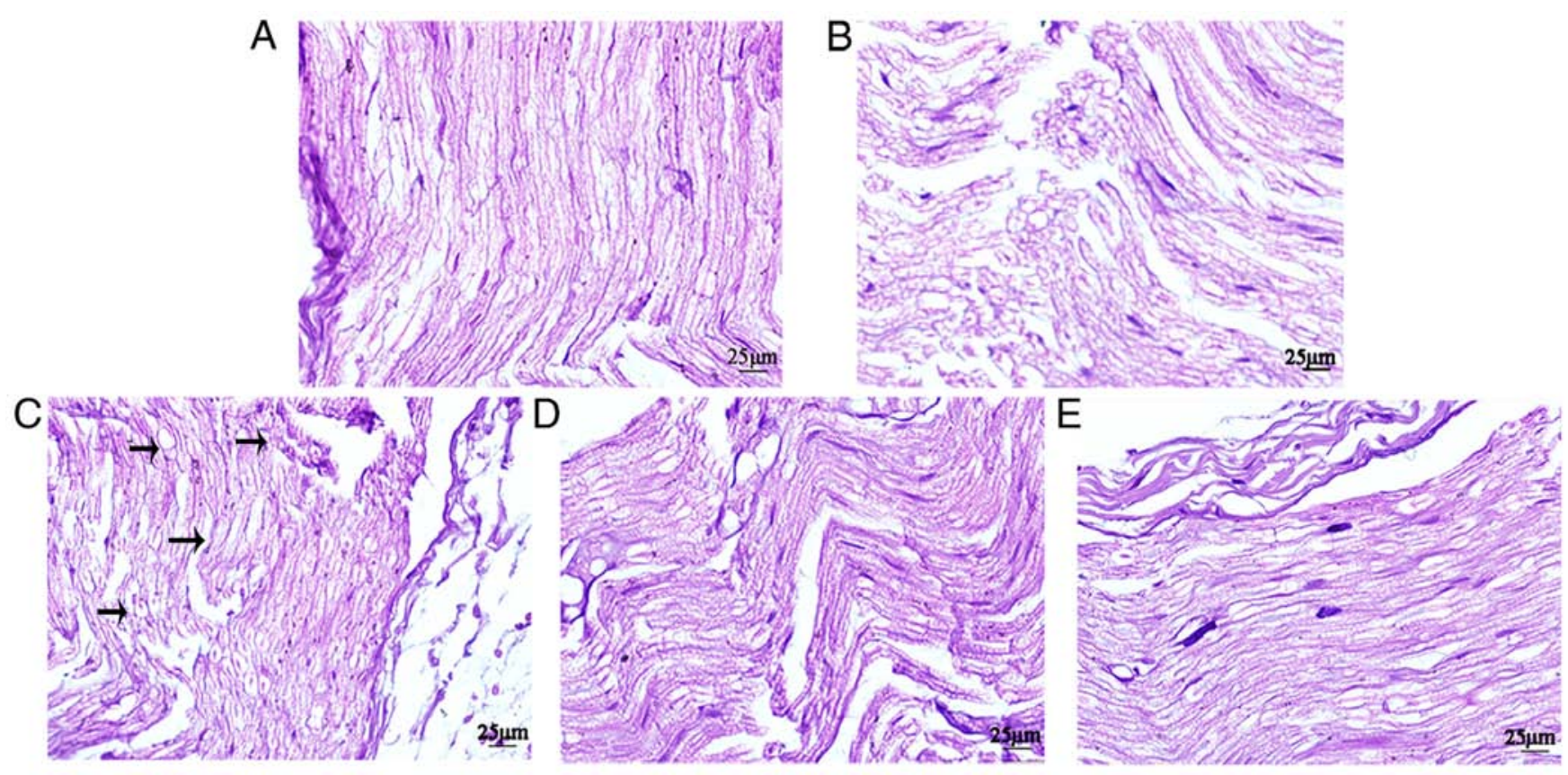

Figure 3. Histopathological alterations in the sciatic nerves of rats from the four groups were examined by conducting hematoxylin and eosin staining ( $\mathrm{n}=8)$. (A) control, (B) sham, (C) ropivacaine, (D) low-dose dexmedetomidine and (E) high-dose dexmedetomidine groups (magnification, $\mathrm{x} 400$ ).

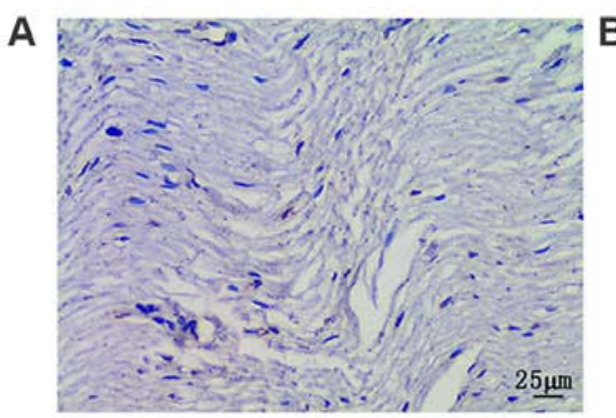

D

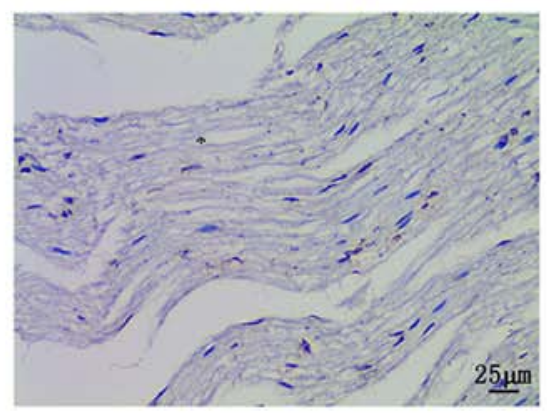

B

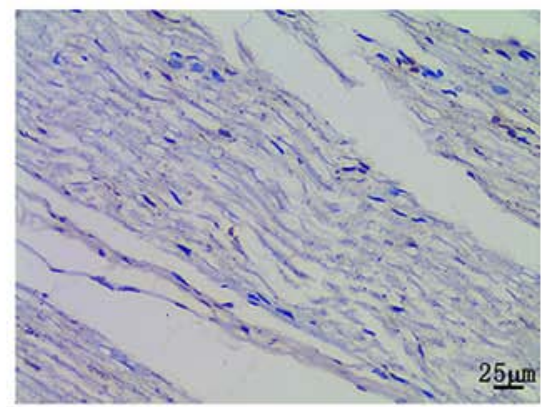

E

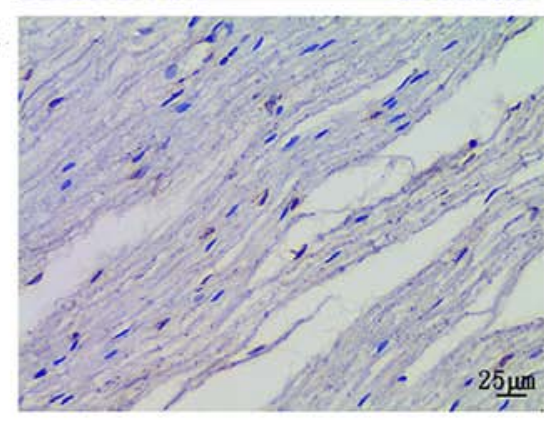

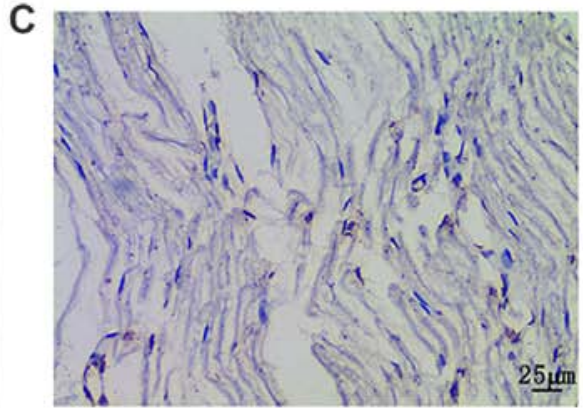

F

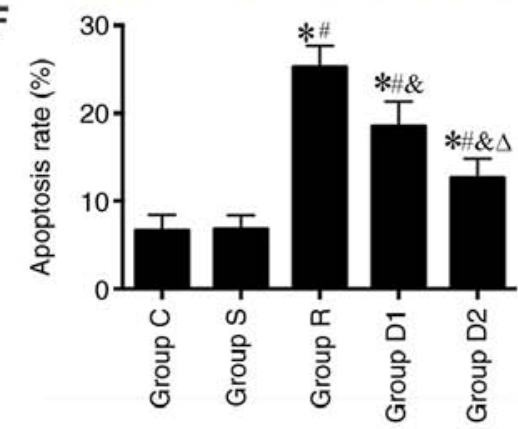

Figure 4. Comparison of the rate of apoptosis in the rats as measured via TUNEL staining (n=8). (A) Control, (B) sham, (C) ropivacaine, (D) low-dose dexmedetomidine and (E) high-dose dexmedetomidine groups (magnification, $\mathrm{x} 400$ ). (F) Quantification of the rate of apoptosis. ${ }^{*} \mathrm{P}<0.05$ vs. group $\mathrm{C}$; ${ }^{\#} \mathrm{P}<0.05$ vs. group $\mathrm{S} ;{ }^{\&} \mathrm{P}<0.05$ vs. group $\mathrm{R} ;{ }^{\Delta} \mathrm{P}<0.05$ vs. group D1. Group $\mathrm{C}$, control group; group $\mathrm{S}$, sham group; group R, ropivacaine group; group D1, low-dose dexmedetomidine group; group D2, high-dose dexmedetomidine group.

results show that the nerve cell apoptosis rate was significantly higher in groups R, D1 and D2 compared with group $\mathrm{S}(\mathrm{P}<0.05)$. Furthermore, the nerve cell apoptosis rate was significantly lower in groups D1 and D2 compared with group $\mathrm{R}(\mathrm{P}<0.05)$. Finally, the nerve cell apoptosis rate was significantly lower in group D2 compared with group D1 $(\mathrm{P}<0.05)$. No significant difference was observed in the nerve cell apoptosis rate between groups $\mathrm{C}$ and $\mathrm{S}$ ( $\mathrm{P}>0.05$; Fig. 4).
Cleaved caspase-3 expression. Alterations in the expression levels of cleaved caspase- 3 were detected via western blotting. The expression of cleaved caspase-3 was significantly higher in group $\mathrm{R}$ compared with groups $\mathrm{S}$ and $\mathrm{C}(\mathrm{P}<0.05)$, and the expression of cleaved caspase-3 was significantly lower in groups D1 and D2 compared with group $\mathrm{R}(\mathrm{P}<0.05)$. In addition, the expression of cleaved caspase-3 was significantly lower in group D2 compared with group D1 $(\mathrm{P}<0.05)$. 

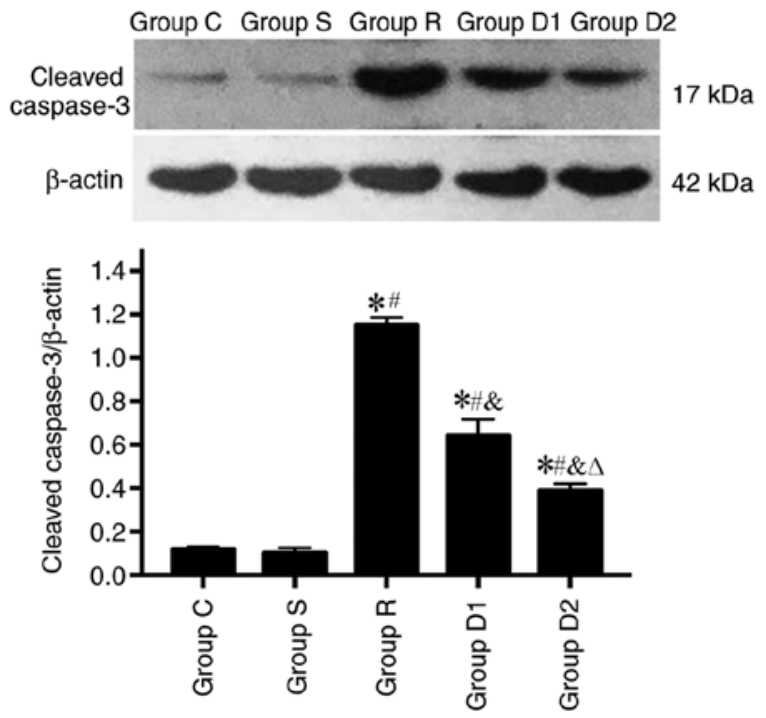

Figure 5. Comparison of the expression of cleaved caspase-3, as measured via western blotting $(n=8)$. " $\mathrm{P}<0.05$ vs. group $\mathrm{C} ;{ }^{\#} \mathrm{P}<0.05$ vs. group $\mathrm{S}$; ${ }^{\text {\& }} \mathrm{P}<0.05$ vs. group $\mathrm{R} ;{ }^{\wedge} \mathrm{P}<0.05$ vs. group D1. Group $\mathrm{C}$, control group; group $\mathrm{S}$, sham group; group R, ropivacaine group; group D1, low-dose dexmedetomidine group group D2, high-dose dexmedetomidine group.

No significant difference was observed in cleaved caspase-3 expression levels between groups $\mathrm{C}$ and $\mathrm{S}$ ( $\mathrm{P}>0.05$; Fig. 5).

\section{Discussion}

In the present study, a rat model of sciatic nerve block was established according to the method described by Kim et al (21). In the study conducted by Kim et al (21), $0.5 \mu \mathrm{g} / \mathrm{kg}$ dexmedetomidine was added to ropivacaine. However, Brummett et al (23) demonstrated that dexmedetomidine added to ropivacaine increases the duration of sensory blockade in a dose-dependent manner. The aforementioned study also identified that the highest dose of dexmedetomidine $(20 \mu \mathrm{g} / \mathrm{kg})$ used in the study displayed the greatest effects, but there were no significant differences in motor function between $(0.5,2.0$ and $6.0 \mu \mathrm{g} / \mathrm{kg}$, respectively) doses of dexmedetomidine. Therefore, in the present study, $20 \mathrm{mg} / \mathrm{kg}$ dexmedetomindin was used in the high dose group and $6.0 \mu \mathrm{g} / \mathrm{kg}$ dexmedetomindin was used in the low dose group. The present study demonstrated that, compared with group S, the PWL of group R was increased, whereas the EPT of group R was decreased, suggesting the successful establishment of a rat model of sciatic nerve block.

To enhance and prolong the anesthetic and analgesic effects of LAs, a variety of LA adjuvants have been investigated, and used in single-shot and continuous peripheral nerve blocks (24-26). $\alpha_{2}$-adrenergic receptors are widely distributed throughout the peripheral and central nervous systems (27). A previous study demonstrated that the use of $\alpha_{2}$-adrenoreceptor agonists in peripheral nerve blocks is safe and beneficial (28). Dexmedetomidine, a potent and highly selective agonist of $\alpha_{2}$-adrenergic receptors, has been used as an analgesic and antinociceptive adjuvant (29).

Previous study has demonstrated that the addition of dexmedetomidine as an adjuvant for nerve block prolongs the duration of analgesia and increases the speed of analgesia onset (30).
The present study indicated that dexmedetomidine prolonged the duration of sciatic nerve block and enhanced the analgesic efficacy of ropivacaine in rats. However, the mechanism underlying $\alpha_{2}$-adrenergic receptor agonist-induced analgesia and sedation remains to be elucidated. A previous study demonstrated that dexmedetomidine binds $\alpha_{2}$-adrenoceptors on the cell membranes of neurons, which leads to the activation of $\mathrm{G}$ protein-coupled inwardly rectifying $\mathrm{K}^{+}$channels and inhibition of $I_{h}$ channels, resulting in hyperpolarization of the membrane (31). By contrast, another study demonstrated that $\alpha_{2}$ agonists produce analgesia and sedation by inhibiting substance $P$ release in the nociceptive pathway at the level of the dorsal root neuron (32). The mechanism underlying $\alpha_{2}$ agonist activity is not fully understood but is probably multifactorial.

In the present study, the addition of dexmedetomidine to ropivacaine for sciatic nerve block shortened sensory and motor block onset time, and extended motor and sensory block duration, particularly sensory duration, compared with ropivacaine alone. When ropivacaine was combined with $6 \mu \mathrm{g} / \mathrm{kg}$ dexmedetomidine, the sensory block duration was prolonged from 150 to $240 \mathrm{~min}$, whereas combination treatment with $20 \mu \mathrm{g} / \mathrm{kg}$ dexmedetomidine prolonged the sensory block duration to $300 \mathrm{~min}$, suggesting that the effect of $20 \mu \mathrm{g} / \mathrm{kg}$ dexmedetomidine was more pronounced compared with $6 \mu \mathrm{g} / \mathrm{kg}$ dexmedetomidine.

Although ropivacaine has been considered a relatively safer LA than bupivacaine in recent years due to its lower degree of motor block and decreased tendency to cause neurotoxicity, a previous study demonstrated that ropivacaine at a clinical concentration $(2.5 \mathrm{mg} / \mathrm{ml})$ is neurotoxic to isolated sensory neurons (33). Ropivacaine can induce the apoptosis of rabbit annulus fibrosus cells in vitro, which involved the mitochondrial signaling pathway (17). The results of the present study suggested that the nerve cell apoptosis rate was increased and the expression of cleaved caspase-3 was upregulated in group $\mathrm{R}$ compared group $\mathrm{S}$, which indicated that ropivacaine induced sciatic nerve toxicity in rats and that the mechanism underlying this effect may be associated with caspase-3-dependent apoptosis.

A previous study has demonstrated that dexmedetomidine can serve as a neuroprotective agent against brain injury via the inhibition of neuronal apoptosis (34). Sun et al (35) identified that dexmedetomidine confers neuroprotection against spinal cord ischemia-reperfusion injury via the suppression of spinal cord inflammation and apoptosis. Another study demonstrated that dexmedetomidine attenuates neuronal injury induced by maternal propofol anesthesia in fetal brains (36). The mechanism underlying dexmedetomidine may be associated with inhibition of propofol-induced caspase-3 activation and the microglial response in fetal brains (37). Kim et al (21) demonstrated that dexmedetomidine added to ropivacaine significantly reduces IL- 6 and IL- $1 \beta$ mRNA levels compared with ropivacaine alone at $60 \mathrm{~min}$ post-intraneural injection. However, the exact effect of dexmedetomidine on the neurotoxicity of LAs is not completely understood. In the present study, the effect of dexmedetomidine on the neurotoxicity of ropivacaine was observed. The results indicated that, compared with group $\mathrm{R}$, the apoptosis rate and caspase- 3 expression levels were significantly reduced in groups D1 and D2. The results of the present study also suggested that dexmedetomidine 
reduced ropivacaine-induced neurotoxicity for sciatic nerve block and that the efficacy of $20 \mu \mathrm{g} / \mathrm{kg}$ dexmedetomidine was greater compared with $6 \mu \mathrm{g} / \mathrm{kg}$ dexmedetomidine.

In conclusion, the present study suggested that dexmedetomidine may serve as a potential LA adjuvant that can prolong the sensory and motor block time of the sciatic nerve, enhance the effect of nerve block and reduce ropivacaine-induced neurotoxicity in rats. Furthermore, the effects of dexmedetomidine were greatest at a dose of $20 \mu \mathrm{g} / \mathrm{kg}$. The results of the present study provided novel insight into the clinical use of dexmedetomidine, suggesting that dexmedetomidine not only provided the longest duration of analgesia but also reduced the neurotoxicity of LAs.

However, the current understanding of the clinical use of dexmedetomidine is not sufficient. In the present study, only two doses were studied, thus, whether further increasing the dose may have a protective effect requires further investigation.

\section{Acknowledgements}

Not applicable.

\section{Funding}

The present study was supported by the National Natural Science Foundation of China (grant no. 81960345) and the Gansu Province Health Industry Plan (grant no. GSWSKY2017-18).

\section{Availability of data and materials}

The datasets used and/or analyzed during the current study are available from the corresponding author on reasonable request.

\section{Authors' contributions}

XX wrote the manuscript. XX, JF and YL conceived and designed the present study. XX, JF, XM, YL, XH and JY performed the experimental procedures. All authors read and approved the final manuscript.

\section{Ethics approval and consent to participate}

The present study was approved by the Medical Ethics Committee of the First Hospital of Lanzhou University (Gansu, China; approval no. LDYYLL2019-111).

\section{Patient consent for publication}

Not applicable.

\section{Competing interests}

The authors declare that they have no competing interests.

\section{References}

1. Scott DB: Local anesthetics: Mechanisms of action and clinica use. Br J Anaesthesia 48: 1031-1032, 1976.

2. Hansen TG: Ropivacaine: A pharmacological review. Expert Rev Neurother 4: 781-791, 2004.
3. Feldman HS and Covino BG: Comparative motor-blocking effects of bupivacaine and ropivacaine, a new amino amide local anesthetic, in the rat and dog. Anesth Analg 67: 1047-1052, 1988.

4. Auyong DB, Cantor DA, Green C and Hanson NA: The effect of fixation technique on continuous interscalene nerve block catheter success: A randomized, double-blind trial. Anesth Analg 124: 959-965, 2017.

5. Rosen MA, Baysinger CL, Shnider SM, Dailey PA, Norton M, Curtis JD, Collins M and Davis RL: Evaluation of neurotoxicity after subarachnoid injection of large volumes of local anesthetic solutions. Anesth Analg 62: 802-808, 1983.

6. Vieira PA, Pulai I, Tsao GC, Manikantan P, Keller B and Connelly NR: Dexamethasone with bupivacaine increases duration of analgesia in ultrasound-guided interscalene brachial plexus blockade. Eur J Anaesthesiol 27: 285-288, 2010.

7. Candido KD, Hennes J, Gonzalez S, Mikat-Stevens M, Pinzur M, Vasic V and Knezevic NN: Buprenorphine enhances and prolongs the postoperative analgesic effect of bupivacaine in patients receiving infragluteal sciatic nerve block. Anesthesiology 113: 1419-1426, 2010.

8. Jarbo K, Batra YK and Panda NB: Brachial plexus block with midazolam and bupivacaine improves analgesia. Can J Anaesth 52: 822-826, 2005.

9. Kaur M and Singh PM: Current role of dexmedetomidine in clinical anesthesia and intensive care. Anesth Essays Res 5: 128-133, 2011.

10. Aksu R and Bicer C: Addition of dexmedetomidine to bupivacaine in supraclavicular brachial plexus block. Clin Invest Med 40: E111-E116, 2017.

11. Ma D Hossain M, Rajakumaraswamy N, Arshad M, Sanders RD, Franks NP and Maze M: Dexmedetomidine produces its neuroprotective effect via the alpha 2A-adrenoceptor subtype. Eur J Pharmacol 502: 87-97, 2004.

12. Perez-Castro R, Patel S, Garavito-Aguilar ZV, Rosenberg A, Recio-Pinto E, Zhang J, Blanck TJ and Xu F: Cytotoxicity of local anesthetics in human neuronal cells. Anesth Analg 108: 997-1007, 2009.

13. Reiz S, Häggmark S, Johansson $G$ and Nath S: Cardiotoxicity of ropivacaine-a new amide local anaesthetic agent. Acta Anaesthesiol Scand 33: 93-98, 1989.

14. Wen X, Liang H, Li H, Ou W, Wang HB, Liu H and Li S: In vitro neurotoxicity by ropivacaine is reduced by silencing Cav3.3 T-type calcium subunits in neonatal rat sensory neurons. Artif Cells Nanomed Biotechnol 46: 1617-1624, 2018.

15. Sun Z, Liu $\mathrm{H}$, Guo Q, Xu X, Zhong $\mathrm{Z}$ and Wang $\mathrm{N}$ : In vivo and in vitro evidence of the neurotoxic effects of ropivacaine: The role of the Akt signaling pathway. Mol Med Rep 6: 1455-1459, 2012.

16. Zhang AZ, Ficklscherer A, Gülecyüz MF, Paulus AC, Niethammer TR, Jansson V and Müller PE: Cell toxicity in fibroblasts, tenocytes, and human mesenchymal stem cells-a comparison of necrosis and apoptosis-inducing ability in ropivacaine, bupivacaine, and triamcinolone. Arthroscopy 33: 840-848, 2017.

17. Cai XY, Xia Y, Yang SH, Liu XZ, Shao ZW, Liu YL, Yang W and Xiong LM: Ropivacaine- and bupivacaine-induced death of rabbit annulus fibrosus cells in vitro: Involvement of the mitochondrial apoptotic pathway. Osteoarthritis Cartilage 23: 1763-1775, 2015.

18. Dhir S, Ganapathy S, Lindsay P and Athwal GS: Case report: Ropivacaine neurotoxicity at clinical doses in interscalene brachial plexus block. Can J Anaesth 54: 912-916, 2007.

19. Werdehausen R, Fazeli S, Braun S, Hermanns H, Essmann F, Hollmann MW, Bauer I and Stevens MF: Apoptosis induction by different local anaesthetics in a neuroblastoma cell line. $\mathrm{Br}$ J Anaesth 103: 711-718, 2009.

20. Yu ZY, Geng J, Li ZQ, Sun YB, Wang SL, Masters J, Wang DX, Guo XY, Li M and Ma D: Dexmedetomidine enhances ropivacaine-induced sciatic nerve injury in diabetic rats. $\mathrm{Br}$ J Anaesth 122: 141-149, 2019.

21. Kim BS, Choi JH, Baek SH and Lee DH: Effects of intraneural injection of dexmedetomidine in combination with ropivacaine in rat sciatic nerve block. Reg Anesth Pain Med 43: 378-384, 2018.

22. Thalhammer JG, Vladimirova M, Bershadsky B and Strichartz GR: Neurologic evaluation of the rat during sciatic nerve block with lidocaine. Anesthesiology 82: 1013-1025, 1995.

23. Brummett CM, Padda AK, Amodeo FS, Welch KB and Lydic R: Perineural dexmedetomidine added to ropivacaine causes a dose-dependent increase in the duration of thermal antinociception in sciatic nerve block in rat. Anesthesiology 111: $1111-1119,2009$. 
24. Candido KD, Winnie AP, Ghaleb AH, Fattouh MW and Franco CD: Buprenorphine added to the local anesthetic for axillary brachial plexus block prolongs postoperative analgesia. Reg Anesth Pain Med 27: 162-167, 2002.

25. Mccartney CJ, Duggan E and Apatu E: Should we add clonidine to local anesthetic for peripheral nerve blockade? A qualitative systematic review of the literature. Reg Anesth Pain Med 32: 330-338, 2007.

26. Parrington SJ, O'Donnell D, Chan VW, Brown-Shreves D, Subramanyam R, Qu M and Brull R: Dexamethasone added to mepivacaine prolongs the duration of analgesia after supraclavicular brachial plexus blockade. Reg Anesth Pain Med 35 422-426, 2010

27. Rosin DL: Distribution of alpha 2A - and alpha 2C -adrenergic receptor immunoreactivity in the central nervous system. Methods Mol Biol 126: 475-505, 2000.

28. Singelyn FJ, Gouverneur JM and Robert A: A minimum dose of clonidine added to mepivacaine prolongs the duration of anesthesia and analgesia after axillary brachial plexus block. Reg Anesth 83: 1046-1050, 1996.

29. Nie Y, Tu W, Shen X, Yu W, Yu Y, Song X, Wang S, Luo A, Cao M, Wu X and Huang S: Dexmedetomidine added to sufentanil patient-controlled intravenous analgesia relieves the postoperative pain after cesarean delivery: A prospective randomized controlled multicenter study. Sci Rep 8: 9952, 2018.

30. Brummett CM, Norat MA, Palmisano JM and Lydic R: Perineural administration of dexmedetomidine in combination with bupivacaine enhances sensory and motor blockade in sciatic nerve block without inducing neurotoxicity in rat. Anesthesiology 109: 502-511, 2008
31. Yang YC, Meng QT, Pan X, Xia ZY and Chen XD Dexmedetomidine produced analgesic effect via inhibition of HCN currents. Eur J Pharmacol 740: 560-564, 2014.

32. Eisenach JC, De Kock M and Klimscha W: Alpha(2)-adrenergic agonists for regional anesthesia. A clinical review of clonidine (1984-1995). Anesthesiology 85: 655-674, 1996.

33. Williams BA, Hough KA, Tsui BY, Ibinson JW, Gold MS and Gebhart GF: Neurotoxicity of adjuvants used in perineural anesthesia and analgesia in comparison with ropivacaine. Reg Anesth Pain Med 36: 225-230, 2011.

34. Zhang MH, Zhou XM, Cui JZ, Wang KJ, Feng Y and Zhang HA: Neuroprotective effects of dexmedetomidine on traumatic brain injury: Involvement of neuronal apoptosis and HSP70 expression. Mol Med Rep 17: 8079-8086, 2018.

35. Sun Z, Zhao T, Lv S, Gao Y, Masters J and Hao W Dexmedetomidine attenuates spinal cord ischemia-reperfusion injury through both anti-inflammation and anti-apoptosis mechanisms in rabbits. J Transl Med 16: 209, 2018.

36. Wei Y, Hu J, Liang Y, Zhong Y, He D, Qin Y, Li L, Chen J, Xiao Q and Xie Y: Dexmedetomidine pretreatment attenuates propofol-induced neurotoxicity in neuronal cultures from the rat hippocampus. Mol Med Rep 14: 3413-3420, 2016.

37. Li J, Xiong M, Nadavaluru PR, Zuo W, Ye JH, Eloy JD and Bekker A: Dexmedetomidine attenuates neurotoxicity induced by prenatal propofol exposure. J Neurosurg Anesthesiol 28: 51-64, 2015.

(i) (2) This work is licensed under a Creative Commons Attribution-NonCommercial-NoDerivatives 4.0 International (CC BY-NC-ND 4.0) License. 